\title{
One-Pot Multi Component Microwave Assisted Synthesis of 4H-Pyrano [2, 3-c] Pyrazoles in Methanol and their Antibacterial Study
}

\author{
Ganesh N Yallappa ${ }^{1(\mathbb{D})}$, Nagaraja Dasappa ${ }^{1},{ }^{*} \mathbb{D}$, Chandrashekhar $\mathrm{U}^{2(\mathbb{D}}$, Aruna G L $\mathrm{L}^{3}$ \\ 1 Department of PG Studies in Chemistry, Government Science College (VTU-RRC), Chitradurga - 577 501, Karnataka, \\ India; gindi.ny1988@gmail.com (G.N.Y.); nagarajachem.18@gmail.com (N.D.); \\ 2 Department of Chemistry, UBDT College of Engineering (VTU), Davangere - 577002 , Karnataka, India; \\ uchandrashekargan@gmail.com (C.U.); \\ 3 Department of Microbiology, Government Science College, Chitradurga-577 501, Karnataka, India; \\ microarunagl@gmail.com (A.G.L.); \\ * Correspondence: nagarajachem.18@gmail.com (N.D.);
}

Scopus Author ID 57198245513

Received: 5.07.2021; Revised: 25.08.2021; Accepted: 28.08.2021; Published: 4.09.2021

\begin{abstract}
Upon literature studies, pyrano-pyrazoles were found to exhibit the biological activity as antimicrobial agents. So literature employed conventional stirring and reflux condensing to afford different pyrano-pyrazoles. Recent researches adopted the Microwave method, which is a simple and convenient method for organic synthesis. In this work, we reported the synthesis of different $4 \mathrm{H}$-pyrano $[2,3-c]$ pyrazoles via two different methods, such as microwave \& conventional stirring at room temperature. Ethyl acetoacetate, hydrazine hydrate, malanonitrile, and different substituted carbonyl compounds were made to react in the presence of methanol solvent \& potassium ter-butoxide, a base catalyst. The microwave method was adopted for all the reactions and found to be more potent than the conventional method. The prepared compounds were characterized by FT-IR, ${ }^{1} \mathrm{HNMR}$, and ${ }^{13} \mathrm{CNMR}$. These compounds were screened for anti-bacterial activity against Staphylococcus aureus and E. coli by well diffusion method and showed the excellent antibacterial property. Our In vitro test for antibacterial study involves excellent MIC $(\mu \mathrm{g} / \mathrm{ml})$ and zone inhibition $(\mathrm{mm})$ measurements of all synthesized compounds. Microwave irradiation synthesis hastens the reaction and is completed very fast. Potassium ter-butoxide, a base catalyst, is proved to be a good catalyst for accelerating the reactions. The yield of all synthesized compounds was good. Different 4H-pyrano [2, 3-c] pyrazoles exhibited good potency against Staphylococcus aureus and E. coli. In the presence of a base catalyst, the reactions are faster than in the absence of a catalyst. The microwave method is a good methodology for the green syntheses. Derivatives of 4H-pyrano [2, 3-c] pyrazoles exhibited excellent MIC $(\mu \mathrm{g} / \mathrm{ml})$ and proved to be as good antibacterial agents.
\end{abstract}

Keywords: 4H-pyrano [2, 3-c] pyrazoles; microwave method; potassium ter-butoxide; methanol solvent; in vitro antibacterial screening; minimum inhibition concentration.

(C) 2021 by the authors. This article is an open-access article distributed under the terms and conditions of the Creative Commons Attribution (CC BY) license (https://creativecommons.org/licenses/by/4.0/).

\section{Introduction}

Upon studying literature, so many heterocycles have featured characteristics in the medicinal field [1]. Over the past decades, these category molecules have been emerging new trends day by day. Recently they have made it more adventurous for the chemists to involve in the research. We studied pyrazoles and made it more interesting to participate in their 
medicinal characters [2-4]. Pyrano [2,3-c] pyrazoles have various Pharmacological applications such as antiviral, anticancer, anti-microbial and so on [5, 6]. The microwave method could reach the green chemistry phenomenon.

In this work, 4H-Pyrano [2, 3-c] Pyrazole molecules were synthesized under microwave irradiation and conventional stirring at room temperature. Potassium ter-butoxide, a strong base, catalyzed the reaction in the presence of methanol as a solvent. Both conventional and microwave methods were compared on their excellence of the reaction. The microwave method was found to be better potent than conventional.

\section{Materials and Methods}

\subsection{Materials used.}

Required chemicals were purchased by SDFCL Company and facilitated by Dept. of Chemistry, Government Science College, Karntaka, India. The melting point was determined into pen capillary tubes in the Buchi B-540 melting point apparatus. The reaction was monitored by thin-layer chromatography using silica gel glass plates. The reaction was visualized by a short Ultraviolet lamp \& isolated in an iodine chamber. FT-IR spectrometer (Vertex series from Bruker), ${ }^{1} \mathrm{H}$ NMR $(400 \mathrm{MHz}) \&{ }^{13} \mathrm{C}$ NMR (100MHz) were used. Glass Petri plates for MIC study were used.

2.1.1. General Procedure for 4H-Pyrano [2, 3-c] Pyrazoles synthesis.

A mixture of Ethyl acetoacetate $(10 \mathrm{Mmol})$, hydrazine hydrate (10 Mmol), malanonitrile $(10 \mathrm{Mmol})$, and Aromatic aldehydes was taken in a flask. A catalytic amount of Potassium t-butoxide $(10 \mathrm{mmol})$ in the presence of methanol solvent $(25-30 \mathrm{ml})$ was added to the mixture and allowed for microwave irradiation. The reaction progressed was monitored by TLC (Pet. Ether: Ethyl acetate). The precipitate thus formed was filtered off, washed with hot water.

The crude solid was then recrystallized by hot ethanol many times to afford pure Pyrano-pyrazoles and finally dried.

\subsection{Antibacterial assay.}

All the compounds synthesized in the present work were screened for antibacterial activity against Staphylococcus aureus, and E. coli by well diffusion method (Kirby-Bauer method) (Çiğdem Eda Balkan et al., 2016) [7, 8]. The bacteria cultures were collected from Vasavi diagnostic laboratory and sub-cultured on nutrient agar slants. They were pre-cultured on nutrient broth overnight and incubated at $37^{\circ} \mathrm{C}$. The culture broths were centrifuged at 1000 rpm for 5minutes; bacterial pellet was suspended in double-distilled sterile water.

In this assay, a concentration of $1 \mathrm{mg} / \mathrm{ml}$ of synthesized compounds was placed into respective wells cut in nutrient agar plates inoculated with test bacteria. Similarly, reference antibiotics streptomycin and ampicillin and solvent dimethyl sulphoxide were also placed into their respective wells. All the plates were incubated at $37^{\circ} \mathrm{C}$ for 24 hours. After incubation, the plates were observed for the inhibition zone surrounding the well, and its diameter was measured. 
6-amino-3-methyl-4-phenyl-2,4-dihydropyrano[2,3-c]pyrazole-5-carbonitrile (5a): White solid, M.P 245-2460 ${ }^{\circ} \mathrm{C}$, yield (\%): 85.00; FT-IR: $v \mathrm{~cm}^{-1} 3374$ (-NH stretch, weak), 3172 ($\mathrm{NH}_{2}$ stretch, weak), 3023 (Ar-H, strong), 2200 (-CN, medium), 1650 (C=C stretch), 1612 $\left(\left(\mathrm{C}=\mathrm{C}\right.\right.$, Pyrazole ring); ${ }^{1} \mathrm{H}-\mathrm{NMR}$ (DMSO, 300MHz): $1.7(3 \mathrm{H}, \mathrm{s}), 2.4-3.2(3 \mathrm{H}, \mathrm{s}), 4.5(1 \mathrm{H}, \mathrm{s})$, $6.8(2 \mathrm{H}, \mathrm{dtd}, \mathrm{J}=7.9,1.3,0.6 \mathrm{~Hz}), 7.1$ (dddd, J= 7.9, 7.7, 1.9, 0.6 Hz), $7.1(\mathrm{tt}, \mathrm{J}=7.7,1.3 \mathrm{~Hz})$,

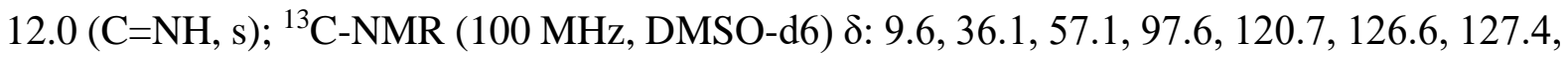
128.3, 135.5, 144.4, 154.7, 160.8; Elemental analysis: calculated for $\mathrm{C}_{14} \mathrm{H}_{12} \mathrm{~N}_{4} \mathrm{O}(\mathrm{C}, \mathrm{H}, \mathrm{N})$ $66.65,4.79,22.21$, Found: 66.66, 4.77, 22.20.

6-amino-4-(2-chlorophenyl)-3-methyl-2,4-dihydropyrano[2,3-c]pyrazole-5-carbonitrile(5b):

Brown solid, M.P 246-248 ${ }^{\circ} \mathrm{C}$, yield (\%): 84.00; FT-IR: $\mathrm{v} \mathrm{cm}^{-1} 3392$ (-NH stretch, weak), 3169 ( $-\mathrm{NH}_{2}$ stretch, weak), 2190(-CN, medium), 1654 (C=C stretch), 1611 ((C=C, Pyrazole ring), 1490 (Ar-C-C stretch, medium), 1053 (C-Cl, stretch, strong), 760 (C-Cl).

6-amino-3-methyl-4-(3-nitrophenyl)-2,4-dihydropyrano[2,3-c]pyrazole-5-carbonitrile(5c):

Dark Yellow solid, M.P 218-220 ${ }^{\circ}$ C, yield (\%): 80.00; FT-IR: $v \mathrm{~cm}^{-1} 3356$ (-NH stretch, weak), 3230 (-NH 2 stretch, weak), 3062 (Ar-H stretch, strong) 2189 (-CN stretch, medium), 1691 (C=N, bend), 1600 (Ar-C-C, Pyrazole ring), 1495 ( $\mathrm{N}=\mathrm{O}$, stretch, Strong), $1453\left(\mathrm{CH}_{2} \& \mathrm{CH}_{3}\right.$, strong); ${ }^{1} \mathrm{H}-\mathrm{NMR}$ (DMSO, 300MHz): 2.1-2.5 (3H, s), 3.3(1H, s), 6.5-7.4 (1H, ddd, J= 8.4, 8.0, $0.4 \mathrm{~Hz}), 7.7(1 \mathrm{H}, \mathrm{ddd}, \mathrm{J}=8.0,1.5,1.4 \mathrm{~Hz}), 8.1-8.2$ ( $2 \mathrm{H}, 7.8$ (dddd, J= 1.7, 1.5, $0.4 \mathrm{~Hz}), 7.8$ (ddd, J=8.4, 1.7, $1.4 \mathrm{~Hz}),(\mathrm{C}=\mathrm{NH}, \mathrm{s}) .{ }^{13} \mathrm{C}-\mathrm{NMR}$ (100 MHz, DMSO-d6) $\delta: ~ 12.6, ~ 18.5, ~ 55.9$, 99.9, 119.3, 125.5, 127.1, 127.8, 128.5, 142.3, 143.1, 144.2, 144.6, 147.5, 159.1; Elemental analysis: calculated for $\mathrm{C}_{14} \mathrm{H}_{11} \mathrm{~N}_{5} \mathrm{O}_{3}(\mathrm{C}, \mathrm{H}, \mathrm{N})$ 56.56, 3.73, 23.56, Found: 56.58, 3.70, 23.52 . 6-amino-4-(4-methoxyphenyl)-3-methyl-2,4-dihydropyrano[2,3-c]pyrazole-5-

carbonitrile $(5 d)$ : Pale Yellow solid, M.P 210-2110 ${ }^{\circ}$ C, yield (\%): 79.00; FT-IR: $v \mathrm{~cm}^{-1} 3363$ ($\mathrm{NH}$ stretch, strong), 3308 (- $\mathrm{NH}_{2}$ stretch, strong), 3062 (Ar-H stretch, strong) 2193 (-CN stretch, medium), 1648 (C=N, bend), 1594 (C-O, stretch), 1486 (Ar-C-C, stretch, medium), 1426 (ArC-C, stretch); ${ }^{1} \mathrm{H}-\mathrm{NMR}$ (DMSO, 300MHz): 1.807 3H,s), 2.481-3.301 (3H, s), $4.47(1 \mathrm{H}, \mathrm{s})$, $6.60(2 \mathrm{H}, \mathrm{ddd}, \mathrm{J}=8.8,1.2,0.6 \mathrm{~Hz}), 7.10(2 \mathrm{H}, \mathrm{ddd}, \mathrm{J}=8.8,1.0,0.6 \mathrm{~Hz}), 12.05(\mathrm{C}=\mathrm{NH}) ;{ }^{13} \mathrm{C}-$ NMR (100 MHz, DMSO-d6) $\delta: 12.33,29.86,55.66,57.28,99.95,111.47,119.17,119.80$, 20.84, 125.03, 128.43, 129.12, 130.66, 142.42, 144.20, 144.99, 147.27, 156.64, 159.76; Elemental analysis: calculated for $\mathrm{C}_{15} \mathrm{H}_{14} \mathrm{~N}_{4} \mathrm{O}_{2}(\mathrm{C}, \mathrm{H}, \mathrm{N}) 63.82,5.00,19.85$, Found: 63.78, $5.05,19.82$.

6-amino-4-[4-(dimethylamino)phenyl]-3-methyl-2,4-dihydropyrano[2,3-c]pyrazole-5carbonitrile(5e): Light Brown solid, M.P 274-276 ${ }^{\circ}$ C, yield (\%): 90.00; FT-IR: $v \mathrm{~cm}^{-1} 3454$ ($\mathrm{NH}$ stretch, strong), 3211 (- $\mathrm{NH}_{2}$ stretch, strong), 3062 (Ar-H stretch, strong) 2203 (-CN stretch, medium), $1634\left(\mathrm{C}=\mathrm{N}\right.$, bend), 1508 (C-O, stretch), $1352\left(\mathrm{CH}_{3} \& \mathrm{CH}_{2}\right.$, bend); ${ }^{1} \mathrm{H}-\mathrm{NMR}$ (DMSO, $300 \mathrm{MHz}): 2.0(3 \mathrm{H}, \mathrm{s}), 2.4-2.4(3 \mathrm{H}, \mathrm{s}), 4.4-4.6(1 \mathrm{H}, \mathrm{s}), 6.5-6.6(2 \mathrm{H}, \mathrm{ddd}, \mathrm{J}=8.2,1.3,0.5 \mathrm{~Hz})$, 7.5-7.8 (2H,ddd, J= 8.2, 1.1, 0.5 Hz), $12.0(\mathrm{C}=\mathrm{NH}) .{ }^{13} \mathrm{C}-\mathrm{NMR}(100 \mathrm{MHz}, \mathrm{DMSO}-\mathrm{d} 6) \delta$ : 9.7, 35.3, 57.9, 98.1, 112.2, 120.9, 127.9, 132.0, 135.4, 149.1, 154.7, 160.5; Elemental analysis: calculated for $\mathrm{C}_{16} \mathrm{H}_{17} \mathrm{~N}_{5} \mathrm{O}(\mathrm{C}, \mathrm{H}, \mathrm{N})$ 65.07, 5.80, 23.71, Found: 65.08, 5.78, 23.70.

6-amino-4-(3-hydroxyphenyl)-3-methyl-2,4-dihydropyrano[2,3-c]pyrazole-5-carbonitrile(5f): Pale Yellow solid, M.P 209-210 C, yield (\%): 89.00; FT-IR: $v \mathrm{~cm}^{-1} 3363$ (Ar-OH, strong), 2216 (CN, medium), 1619 (C=N, bend), 1602 (Ar-C-C, stretch); ${ }^{1} \mathrm{H}-\mathrm{NMR}$ (DMSO, 300MHz): 2.4-2.4 (3H, s), 2.4-3.3 (3H, s), $7.0(1 \mathrm{H}, \mathrm{dd}, \mathrm{J}=10.1,9.5 \mathrm{~Hz}), 7.0-7.7(3 \mathrm{H}, 7.4$ (tt, J=7.3, 1.5 $\mathrm{Hz}$ ), 7.7 (dddd, J= 7.9, 7.3, 2.0, $0.4 \mathrm{~Hz})$ ), $7.7(2 \mathrm{H}$, dddd, J= 7.9, 1.5, 1.5, 0.4 Hz), $7.8(1 \mathrm{H}, \mathrm{d}$, $\mathrm{J}=9.5 \mathrm{~Hz}), 8.6(1 \mathrm{H}, \mathrm{d}, \mathrm{J}=10.1 \mathrm{~Hz}) .{ }^{13} \mathrm{C}-\mathrm{NMR}(100 \mathrm{MHz}, \mathrm{DMSO}-\mathrm{d}) \delta: 9.7,36.1,57.2,97.6$, 
113.7, 114.0, 118.1, 120.7, 129.2, 135.5, 145.9, 154.7, 157.3, 160.8; Elemental analysis: calculated for $\mathrm{C}_{14} \mathrm{H}_{12} \mathrm{~N}_{4} \mathrm{O}_{2}(\mathrm{C}, \mathrm{H}, \mathrm{N})$ 62.68, 4.51, 20.88, Found: 62.61, 4.39, 20.90.

6-amino-4-(4-hydroxyphenyl)-3-methyl-2,4-dihydropyrano[2,3-c]pyrazole-5-carbonitrile (5g): Brown solid, M.P. 192-194 C, solid, yield (\%) 80.00; FT-IR: v cm-1 3363 (Ar-OH, strong), 2216 (CN, medium), $1619\left(\mathrm{C}=\mathrm{N}\right.$, bend), 1602 (Ar-C-C, stretch); ${ }^{1} \mathrm{H}-\mathrm{NMR}$ (DMSO, 300MHz): 2.4-2.4 (3H, s), 2.4-3.3 (3H, s), $7.0(1 \mathrm{H}, \mathrm{dd}, \mathrm{J}=10.1,9.5 \mathrm{~Hz}), 7.0-7.7(3 \mathrm{H}, 7.4$ (tt, $\mathrm{J}=7.3,1.5 \mathrm{~Hz}$ ), 7.7 (dddd, J= 7.9, 7.3, 2.0, $0.4 \mathrm{~Hz})$ ), $7.7(2 \mathrm{H}$, dddd, J= 7.9, 1.5, 1.5, $0.4 \mathrm{~Hz}), 7.8$ $(1 \mathrm{H}, \mathrm{d}, \mathrm{J}=9.5 \mathrm{~Hz}), 8.6(1 \mathrm{H}, \mathrm{d}, \mathrm{J}=10.1 \mathrm{~Hz}) .{ }^{13} \mathrm{C}-\mathrm{NMR}(100 \mathrm{MHz}, \mathrm{DMSO}-\mathrm{d} 6)$ ) $\delta: 9.7,35.7,55.5$, 57.5, 97.8, 111.5, 115.3, 119.7, 120.8, 135.3, 135.5, 145.1, 147.2, 154.6, 160.4; Elemental analysis: calculated for $\mathrm{C}_{14} \mathrm{H}_{12} \mathrm{~N}_{4} \mathrm{O}_{2}(\mathrm{C}, \mathrm{H}, \mathrm{N})$ 62.68, 4.51, 20.88, Found: 62.70, 4.48, 20.87 6-amino-4-(2,4-dihydroxyphenyl)-3-methyl-2,4-dihydropyrano[2,3-c]pyrazole-5-carbonitrile (5h): Yellow solid, M.P. 200-202 ${ }^{\circ}$, yield (\%) 82.00; FT-IR: $\mathrm{v} \mathrm{cm}^{-1} 3363$ (Ar-OH, strong), 2216 (CN, medium), 1619 (C=N, bend), 1602 (Ar-C-C, stretch); ${ }^{1} \mathrm{H}-\mathrm{NMR}$ (DMSO, 300MHz): 2.4-2.5 (3H, s), 2.4-3.3 (3H, s), $7.0(1 \mathrm{H}, \mathrm{dd}, \mathrm{J}=10.1,9.5 \mathrm{~Hz}), 7.0-7.7(3 \mathrm{H}, 7.4(\mathrm{tt}, \mathrm{J}=7.3,1.5$ $\mathrm{Hz}), 7.7$ (dddd, J= 7.9, 7.3, 2.0, $0.4 \mathrm{~Hz})$ ), $7.7(2 \mathrm{H}$, dddd, J= 7.9, 1.5, 1.5, 0.4 Hz), $7.8(1 \mathrm{H}, \mathrm{d}$, $\mathrm{J}=9.5 \mathrm{~Hz}), 8.6(1 \mathrm{H}, \mathrm{d}, \mathrm{J}=10.1 \mathrm{~Hz})$. Elemental analysis: calculated for $\mathrm{C}_{14} \mathrm{H}_{13} \mathrm{~N}_{4} \mathrm{O}_{3}(\mathrm{C}, \mathrm{H}, \mathrm{N})$ 58.94, 4.56, 19.64, Found: 58.90, 4.58, 19.62.

6-amino-4-[(E)-2-phenylethenyl]-2,4-dihydropyrano[2,3-c]pyrazole-5-arbonitrile

$(5 i)$ : Yellow solid, M.P 269-271 ${ }^{\circ} \mathrm{C}$, yield (\%): 84.00; FT-IR: $v \mathrm{~cm}^{-1} 3345$ (-NH, stretch, strong), $3227\left(\mathrm{NH}_{2}\right.$ stretch, strong), $3027(\mathrm{CH}=\mathrm{CH}$ stretch) $2215(\mathrm{CN}$, medium),1619 (C=N, bend), 1602 (Ar-C-C, stretch); ${ }^{1} \mathrm{H}-\mathrm{NMR}$ (DMSO, 300MHz): 1.0-1.2 (3H, s), 2.0-2.5 (3H, s), 3.9-4.9 $(1 \mathrm{H}, \mathrm{d}, \mathrm{J}=4.4 \mathrm{~Hz}), 6.6-6.7(2 \mathrm{H}, 6.8(\mathrm{~d}, \mathrm{~J}=17.1 \mathrm{~Hz}), 7.2(\mathrm{dd}, \mathrm{J}=17.1,4.4 \mathrm{~Hz})), 7.5(1 \mathrm{H}, \mathrm{s}), 7.5$ $(1 \mathrm{H}, \mathrm{tt}, \mathrm{J}=7.7,1.3 \mathrm{~Hz}), 7.8-7.9(4 \mathrm{H}, 7.3$ (dddd, J=7.7, 7.6, 1.8, 0.5 Hz), 8.0-8.9 (dddd, J=7.6, 1.8, 1.3, 0.5 Hz). ${ }^{13} \mathrm{C}-\mathrm{NMR}(100 \mathrm{MHz}$, DMSO-d6 ) $\delta: 10.4,35.9,57.1,97.7,120.8,126.8$, 127.6, 135.6, 142.7, 154.7, 160.8; Elemental analysis: calculated for $\mathrm{C}_{17} \mathrm{H}_{13} \mathrm{~N}_{4} \mathrm{O}(\mathrm{C}, \mathrm{H}, \mathrm{N})$ 70.58, 4.49, 19.37, Found: 70.57, 4.50, 19.42 .

6-amino-4-(furan-2-yl)-1,3a,4,7a-tetrahydropyrano[2,3-c]pyrazole-5-carbonitrile

$(5 j)$ : Brown solid, M.P 255-256 ${ }^{\circ}$, yield (\%): 80.00; FT-IR: $v \mathrm{~cm}^{-1} 3329$ (-NH, stretch, strong), 3220 ( $\mathrm{NH}_{2}$ stretch, strong), 2214 (CN, medium), 1595 (CO, stretch, furan ring), 1602 (Ar-C-C, stretch), 1453 (CH3 strong); ${ }^{1} \mathrm{H}-\mathrm{NMR}$ (DMSO, 300MHz): 1.2 (3H, s), 2.0-2.5 (3H, s), 3.3-3.9 $(1 \mathrm{H}, \mathrm{d}, \mathrm{J}=4.4 \mathrm{~Hz}), 6.2-6.6(2 \mathrm{H}, 6.8(\mathrm{~d}, \mathrm{~J}=17.1 \mathrm{~Hz}), 7.0(\mathrm{dd}, \mathrm{J}=17.1,4.4 \mathrm{~Hz})), 7.5(1 \mathrm{H}, \mathrm{s}), 7.3$ $(1 \mathrm{H}, \mathrm{tt}, \mathrm{J}=7.7,1.3 \mathrm{~Hz}), 7.6-7.9(4 \mathrm{H}, 7.3$ (dddd, J=7.7, 7.6, 1.8, 0.5 Hz), 7.9-8.0 (dddd, J=7.6, $1.8,1.3,0.5 \mathrm{~Hz}) .{ }^{13} \mathrm{C}-\mathrm{NMR}(100 \mathrm{MHz}, \mathrm{DMSO}-\mathrm{d} 6) \delta: 9.6,31.3,57.5,97.5,120.5,124.3,124.9$, 126.4, 136.0, 149.7, 154.2, 160.6; Elemental analysis: calculated for $\mathrm{C}_{12} \mathrm{H}_{10} \mathrm{~N}_{4} \mathrm{O}_{2}(\mathrm{C}, \mathrm{H}, \mathrm{N})$ 59.50, 4.16, 23.13, Found: 59.48, 4.13, 23.11.

\section{Results and Discussion}

The synthesized compounds exhibited FT-IR ranges between $3300-3450 \mathrm{~cm}^{-1}$ and $2200-2230 \mathrm{~cm}^{-1}$ confirmed $-\mathrm{NH}$ of pyrazole ring and $\mathrm{CN}$ group, respectively. Likewise, ${ }^{1} \mathrm{H}-$ NMR spectra showed the range 6.5-8.0 ppm and 8.0-12.1 ppm which confirmed the aromatic protons of pyrazoles and -NH of pyrazole, respectively. Reddy M B et al. reported the synthesis of Pyrazolo [2,3-c] pyrazoles by the catalyst imidazole by conventional method [9].

This work consumes more reaction time as well as a lower yield of products. Khurana $\mathrm{J}$ M, et al. reported the work on Pyrazolo [2,3-c] pyrazoles synthesis in the presence of an Ionic liquid [bmim] $\mathrm{OH}$ under solvent-free conditions. This work revealed the reaction time of all the reactions at approx. 5 mins [10]. We studied some literature on the synthesis of Pyrano [2, 
3-c] pyrazoles by different methods. These researches only revealed the synthesis of compounds by convenient methods but not focussed on shorter reaction times and the yield [11-15].

We are herein reported that reactions under microwave irradiation were completed faster than the convention method $(<5 \mathrm{~min})$. Meanwhile, compounds yielded excellent under the influence of microwave. $\mathrm{K}-\mathrm{tBuO}$, a catalyst, provoked the reactions in the presence of methanol solvent.

From Table 1, Under microwave, compounds 5a and 5d were found to be completed the reaction at a lesser time ( $>3 \mathrm{mins}$ ) and afforded the yields $85.00 \%$ and $79.00 \%$ respectively. Due to only the Phenyl group without any electron releasing group and electron-withdrawing group attached to the Pyrano-pyrazole moiety, the yield of 5a compound is excellent compared to $5 \mathrm{~d}$. The compounds $5 \mathrm{a}, 5 \mathrm{~b}, 5 \mathrm{~d}, 5 \mathrm{e}, 5 \mathrm{f}, 5 \mathrm{~g}$, and $5 \mathrm{~h}$ possess electron releasing group attached to the phenyl group, completed the reaction within 4 mins and yielded excellent compared to $5 c$, which possess electron-withdrawing group at a phenyl substituent.

Table 1. Synthesis of Pyrano [2,3-c] pyrazoles by Microwave method.

\begin{tabular}{l|c|c|c|c|c} 
Compounds & \multicolumn{2}{|c|}{ Time (min) } & \multicolumn{2}{c}{ Yield (\%) } & M.P ${ }^{\mathbf{0}}$ \\
\hline $\mathbf{5 a}$ & Microwave & Convention & Microwave & Convention & \\
\hline $\mathbf{5 b}$ & 2.5 & $5-6$ & 85.00 & 80.00 & $245-246$ \\
\hline $\mathbf{5 c}$ & 4.5 & $10-12$ & 84.00 & 75.00 & $246-248$ \\
\hline $\mathbf{5 d}$ & 5.0 & 10 & 80.00 & 60.00 & $218-220$ \\
\hline $\mathbf{5 e}$ & $2-3$ & $8-10$ & 83.00 & 62.00 & $210-211$ \\
\hline $\mathbf{5 f}$ & $3-4$ & 12 & 89.00 & 75.00 & $274-276$ \\
\hline $\mathbf{5 g}$ & 4.0 & $11-12$ & 84.00 & 72.00 & $209-210$ \\
\hline $\mathbf{5 h}$ & $3-4$ & 10 & 80.00 & 70.00 & $192-194$ \\
\hline $\mathbf{5 i}$ & 4.0 & $10-11$ & 82.00 & 72.00 & $200-202$ \\
\hline $\mathbf{5 j}$ & 5.0 & 12 & 80.00 & 70.00 & $269-271$ \\
\hline
\end{tabular}

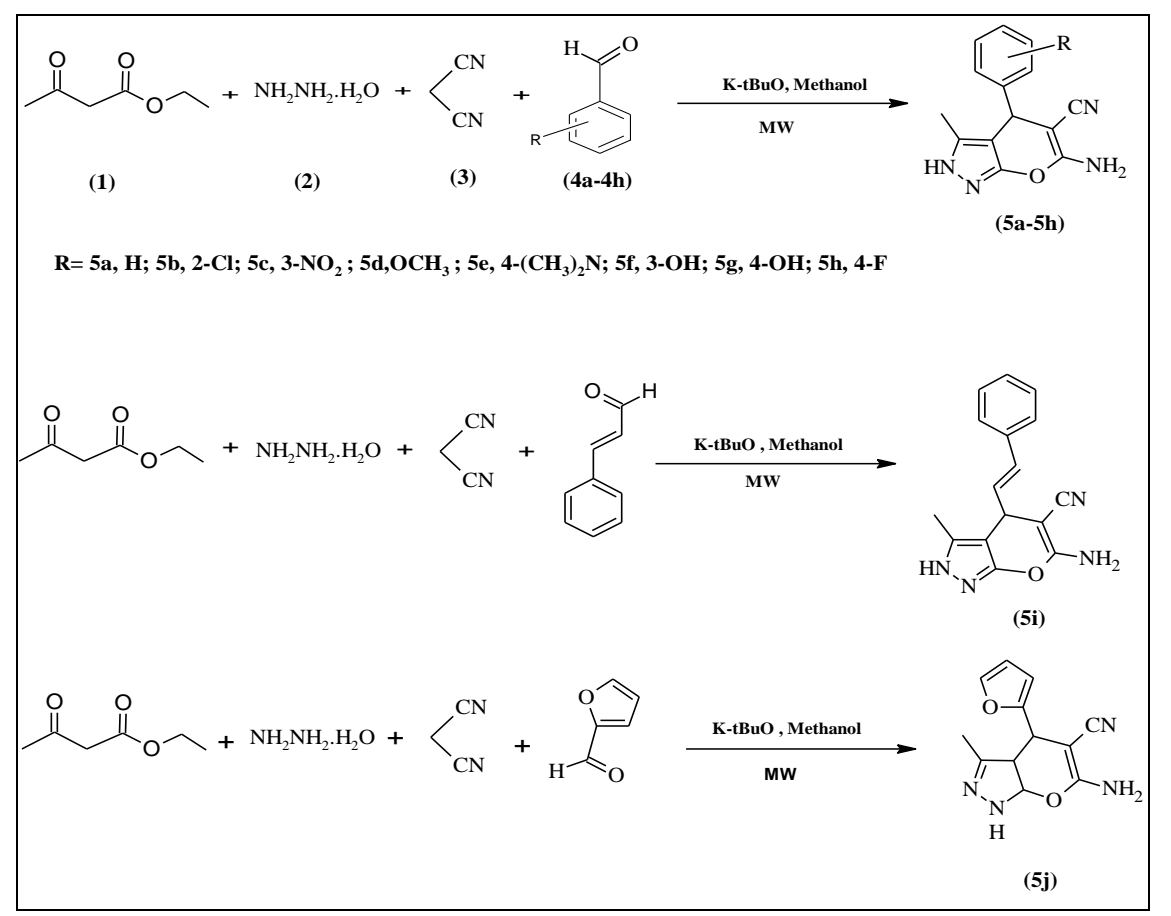

Scheme 1. Base catalysed microwave assisted synthesis of Pyrano[2,3-c]pyrazoles in Methanol solvent.

\subsection{Antibacterial assay.}

After incubation, inhibition zones formed around the wells were measured in millimetres. This study was performed in triplicates. The results showed (in Fig. 1) In vitro 
antibacterial activity of all the synthesized compounds tested at $1 \mathrm{mg} / \mathrm{ml}$ concentration showed low to high activity against Staphylococcus aureus, and E. coli.

Mahavir Parshad et al. in 2015 revealed the study of antibacterial activity of Pyrano [2,3-c] pyrazoles. Zone inhibition and MIC of most synthesized compounds were found to exhibit less activity [16]. We referred to many kinds of literature of antibacterial activity of Pyrano [2,3-c] pyrazoles possess less activity (MIC) [17, 18].

Among the tested compounds and standard antibiotics, relatively $5 \mathrm{~d}$ showed the highest activity (23 $\mathrm{mm})$ against Staphylococcus aureus, and E. coli, which is higher than reference antibiotics (in Fig. 2)

The compound 5i has shown the highest activity against Staphylococcus aureus, which is slightly higher than ampicillin. The activity of compounds 5b and 5j against Staphylococcus aureus is equal to ampicillin. All other compounds showed moderate antibacterial activity. This showed that the compounds synthesized in our work are effective antibacterial activity.

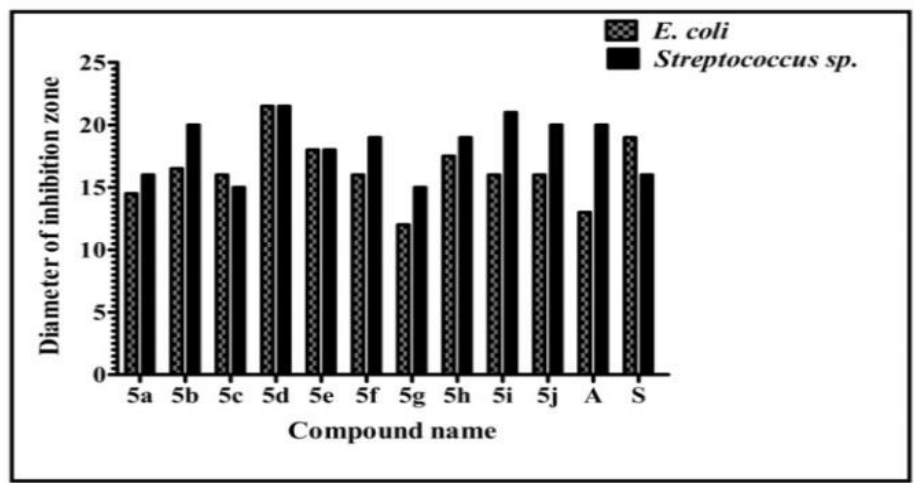

Figure 1. Graph representation for antibacterial activity of test compounds against E. coli and Staphylococcus aureus in comparision with reference antibiotics Ampicillin (A) and Streptomycin (S).

\subsection{Minimum inhibitory concentration of Compounds.}

The compounds $5 \mathrm{~d}, 5 \mathrm{e}$, and $5 \mathrm{~h}$ inhibited $E$. coli growth in lowest $(7.8125 \mu \mathrm{g} / \mathrm{ml})$ inhibitory concentration. The other compounds $5 \mathrm{~d}, 5 \mathrm{f}, 5 \mathrm{~h}, 5 \mathrm{i}$, and $5 \mathrm{j}$ have the lowest $(7.8125$ $\mu \mathrm{g} / \mathrm{ml}$ ) inhibitory concentration against Staphylococcus aureus (Table 2.). This showed that the compounds synthesized in our work have great potential to inhibit+/kill the test bacteria in low concentration.

MIC is defined as the lowest concentration of the antibacterial agent where no visible growth is observed in the test tube (bacteriostatic concentration). The method of Volleka et al. (2001), modified by Usman et al. (2007), was used to determine the MIC of the compounds [19]. The compounds were diluted by double serial dilution method (1-2) to a working concentration ranging from $1000 \mu \mathrm{g} / \mathrm{ml}$ to $7.812 \mu \mathrm{g} / \mathrm{ml}$ using nutrient broth. Later all the test tubes containing nutrient broth and compounds in variable concentration were inoculated with $0.1 \mathrm{ml}$ respective test bacterial suspension. After 18 hours of incubation at $37^{\circ} \mathrm{C}$, the test tubes were observed for growth, and turbidity was determined calorimetrically. The least concentration of the compounds (or highest dilution of compounds) that completely inhibited the growth of the test organism, i.e., where no turbidity was observed, is the minimum inhibitory concentration (MIC) of the compounds. A control experiment was done parallel to check the influence of the solvents alone (without compound) on the growth of the test organisms. Solvents were diluted appropriately with sterile nutrient broth, followed by inoculation of test bacterial suspension and incubation. Positive control was prepared by using 
$2 \mathrm{ml}$ of sterile nutrient broth followed by inoculation of $0.1 \mathrm{ml}$ of test bacterial suspension and incubation (EL-Kamali et al., 2010) [20].

Table 2. Minimum Inhibitory Concentration (MIC).

\begin{tabular}{c|c|c|c}
\multirow{2}{*}{$\begin{array}{c}\text { Sl. } \\
\text { No. }\end{array}$} & \multirow{2}{*}{ Compound name } & \multicolumn{2}{|c}{ MIC $(\boldsymbol{\mu g} / \mathbf{m l})$} \\
\cline { 3 - 4 } $\mathbf{1}$ & & $\boldsymbol{E}$. coli & S. aureus \\
\hline $\mathbf{2}$ & $5 \mathrm{a}$ & 15.625 & 15.625 \\
\hline $\mathbf{3}$ & $5 \mathrm{~b}$ & 15.625 & 31.125 \\
\hline $\mathbf{4}$ & $5 \mathrm{c}$ & 15.625 & 62.25 \\
\hline $\mathbf{5}$ & $5 \mathrm{~d}$ & 7.8125 & 7.8125 \\
\hline $\mathbf{6}$ & $5 \mathrm{e}$ & 7.8125 & 15.625 \\
\hline $\mathbf{7}$ & $5 \mathrm{f}$ & 31.125 & 7.8125 \\
\hline $\mathbf{8}$ & $5 \mathrm{~g}$ & 15.625 & 15.625 \\
\hline $\mathbf{9}$ & $5 \mathrm{~h}$ & 7.8125 & 7.8125 \\
\hline $\mathbf{1 0}$ & $5 \mathrm{i}$ & 31.125 & 7.8125 \\
\hline
\end{tabular}
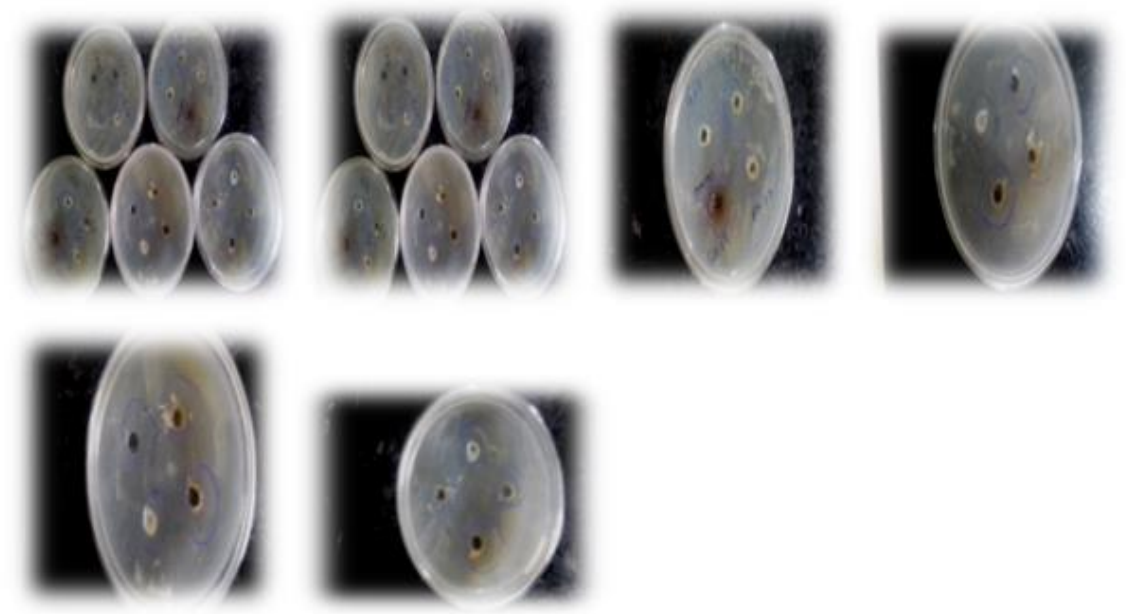

Figure 2. Images: Measurements of Zone Inhibition at $1 \mathrm{mg} / \mathrm{ml}$ concentration of compounds $5 \mathrm{a}-5 \mathrm{j}$ against Staphylococcus aureus, and E. coli.

\section{Conclusions}

We reported that Multicomponent reactions are instant reactions under the microwave. But in the case of convention, the reaction time is high compared to microwave. The yield of all synthesized compounds is excellent in the microwave. Potassium t-butoxide is proved as a promising catalyst. Most of the synthesized compounds showed good activity against Gram+ve and Gram--ve bacteria. Some of the synthesized compounds showed moderate antibacterial activity.

\section{Funding}

This research work has not received any external fund.

\section{Acknowledgments}

We express thanks to all Institutions/Persons/Scholars for the help during our Research work tenure.

\section{Conflicts of Interest}

No Conflicts of interest declared by all the Authors. 


\section{References}

1. Dwivedi, K.D.; Borah, B.; Chowhan, L.R. Ligand Free One-Pot Synthesis of Pyrano[2,3-c]pyrazoles in Water Extract of Banana Peel (WEB): A Green Chemistry Approach. Frontiers in Chemistry 2020, 7, 944952, https://doi.org/10.3389/fchem.2019.00944.

2. Shrivas, P.; Pandey, R.; Zodape, S.; Wankhade, A.; Pratap, U. Green synthesis of pyranopyrazoles via biocatalytic one-pot Knoevenagel condensation-Michael-type addition-heterocyclization cascade in nonaqueous media. Research on Chemical Intermediates 2020, 46, 2805-2816, https://doi.org/10.1007/s11164020-04122-x.

3. Faroughi Niya, H.; Hazeri, N.; Maghsoodlou, M.T. Synthesis and characterization of Fe3O4@THAM-SO3H as a highly reusable nanocatalyst and its application for the synthesis of dihydropyrano[2,3-c]pyrazole derivatives. Applied Organometallic Chemistry 2020, 34, https://doi.org/10.1002/aoc.5472.

4. Sikandar, S.; Zahoor, A.F. Synthesis of pyrano[2,3-c]pyrazoles: A review. Journal of Heterocyclic Chemistry 2021, 58, 685-705, https://doi.org/10.1002/jhet.4191.

5. Insuasty, D.; Castillo, J.; Becerra, D.; Rojas, H.; Abonia, R. Synthesis of Biologically Active Molecules through Multicomponent $\quad$ Reactions. $\quad$ Molecules $\quad \mathbf{2 0 2 0}, \quad 25, \quad 505-576$, https://doi.org/10.3390/molecules25030505.

6. Vasava, M.S.; Bhoi, M.N.; Rathwa, S.K.; Shetty, S.S.; Patel, R.D.; Rajani, D.P.; Rajani, S.D.; Patel, A.; Pandya, H.A.; Patel, H.D. Novel 1,4-dihydropyrano[2,3-c]pyrazole derivatives: Synthesis, characterization, biological evaluation and in silico study. Journal of Molecular Structure 2019, 1181, 383-402, https://doi.org/10.1016/j.molstruc.2018.12.053.

7. Ziarani, G.; Nouri, F.; Rahimifard, M.; Badiei, A.; Abolhasani Soorki, A. One-pot synthesis of pyrano[2,3c]pyrazoles using SBA-15-PR-NH2 and their antimicrobial activities. Revue Roumaine de Chimie 2015, 60, 331-337.

8. Çiğdem, E.B.; Murat, K.; Demet, Ç.; Sabiha, A.; Yalçın, D.; Zeki, Ç. Kafkas. Acute Gastroenteritis Agents Among 0-5 Years-Old Turkish Children. J Med Sci. 2016, 6, 288-294.

9. Reddy, M.B.; Pasha, M.A. One-pot, multicomponent synthesis of $4 H$-Pyrano[2,3-c]pyrazoles in water at $25^{\circ}$ C. Ind.J.Chem. (B) 2012, 3, 537-541.

10. Khurana, J.M.; Chaudhary, A. Efficient and green synthesis of 4H-pyrans and 4H-pyrano[2,3-c] pyrazoles catalyzed by task-specific ionic liquid [bmim] OH under solvent-free conditions. Green Chemistry Letters and Reviews 2012, 5, 633-638, https://doi.org/10.1080/17518253.2012.691183.

11. Tacconi, G.; Gatti, G.; Desimoni, G.; Messori, V. A New Route to 4H-Pyrano[2,3-c]pyrazoles. Journal für Praktische Chemie 1980, 322, 831-834, https://doi.org/10.1002/prac.19803220519.

12. Kiyani, H.; Samimi, H.; Ghorbani, F.; Esmaieli, S. One-pot, four-component synthesis of pyrano [2, 3-c] pyrazoles catalyzed by sodium benzoate in aqueous medium. Current Chemistry Letters 2013, 2, 197-206, https://doi.org//10.5267/j.ccl.2013.07.002.

13. Imene, A.K.; Wassima, G.; Raouf, B.; Taous, B.; Abdelmadjid, D. Phenylboronic acid-catalysed a four component synthesis of Pyrano [2,3-c] pyrazole derivatives in aqueous media: an ecofriendly method. Der Pharma Chemica 2015, 7, 175-180.

14. Yallappa, G.N.; Nagaraja, D.; Chandrashekhar, U. Nano-Catalyzed Green Synthesis Of Pyrazole Derivatives \& Its Biological Activity As Eac Receptor Antagonists. Pharmacophore 2019, 10, 28-32.

15. Elgemeie, G.H.; Elghandour, A.H.; Hussein, A.M. Amino Acids in Heterocyclic Synthesis: Novel Synthesis of Pyrano[2,3-c]pyrazoles, Pyrazolo[3,4-b]pyridine, and Pyrido[1,2-a]benzimidazoles. Synthetic Communications 2004, 34, 3293-3302, https://doi.org/10.1081/SCC-200030553.

16. Parshad, M.; Verma, V.; Kumar, D. Iodine-mediated efficient synthesis of pyrano[2,3-c]pyrazoles and their antimicrobial activity. Monatshefte für Chemie - Chemical Monthly 2014, 145, 1857-1865, https://doi.org/10.1007/s00706-014-1250-5.

17. Addoum, B.; Idiken, M.; Sakoui, S.; Derdak, R.; Aniq Filali, O.; Elmakssoudi, A.; Soukri, A. Synthesis, Characterization of Pyrano-[2, 3-c]-Pyrazoles Derivatives and Determination of Their Antioxidant Activities. Iranian Journal of Toxicology 2021, 15, 175-194.

18. Reddy, G.M.; Garcia, J.R.; Zyryanov, G.V.; Sravya, G.; Reddy, N.B. Pyranopyrazoles as efficient antimicrobial agents: Green, one pot and multicomponent approach. Bioorganic Chemistry 2019, 82, 324331, https://doi.org/10.1016/j.bioorg.2018.09.035.

19. Manzo, L.M.; Bako, H.D.; Idrissa, M. Phytochemical Screening and Antibacterial Activityof Stem Bark, Leaf and Root Extract of Sclerocaryabirrea (A. Rich.) Hochst. 2017, 5, 127-131, https://doi.org/10.15171/ijep.2017.29.

20. El-Kamali, H.H.; El-Amir, M.Y. Antibacterial activity and phytochemical screening of ethanolic extracts obtained from selected Sudanese medicinal plants. Current Research Journal of Biological Sciences 2010, 2, 143-146. 